Article

\title{
Comparison of infrared thermal imaging with two canine pain assessment tools in dogs undergoing treatment for chronic back pain.
}

\author{
Emily Freeman' ${ }^{1}$ Jennifer F. Johnson ${ }^{2}$, John C. Godbold Jr. ${ }^{3}$, Ronald J. Riegel ${ }^{4 *}$ \\ 1 Animal Rehab Center, 178 S Charlotte St, Asheville, NC, 28801 United States, emilyfreeman.pt@gmail.com \\ 2 International Veterinary Academy of Pain Management, PO Box 1868, Mt. Juliet, TN, 37121, United States, \\ jiohnsonvmd@gmail.com \\ 3. Stonehaven Veterinary Consulting, 33 Sunhaven Drive, Jackson, TN, 38305, United States, SVC@earth- \\ link.net \\ 4. Digatherm LLC, 5100 FL-40, Suite 1000, Ocala, FL, United States, aimladvm@aol.com \\ * Correspondence: aimladvm@aol.com
}

\begin{abstract}
Historically, the evaluation and assessment of the clinical response to treatment for canine back pain is subjective and relies on owner and clinician assessment of pain. Sequential infrared thermal images (IRTIs) objectively measure the patient's physiological response after a prescribed series of photobiomodulation therapy (PBMT) treatments. Qualifying participants had histories of pain and dysfunction associated with spinal osteoarthritis or intervertebral disk disease, or of nonspecific uni- or bilateral back pain along the paravertebral epaxial muscles. Each patient was initially IRT imaged prior to PBMT treatment and then received multiple PBMT treatments delivered to the appropriate spinal area on days 1, 2, 3, and 4. Participants were reimaged on day 7. IRT images provided an objective assessment of the physiologic status of each patient after the PBMT regimen. The thermal changes in IRT images correlated with statistically significant changes in Colorado State University Canine Chronic Pain Scale scoring (CSU-CCPS) and owner assessment using the Canine Brief Pain Inventory (CBPI), which includes a Pain Severity Score (CBPI-PS) and Pain Interference Score (CBPI-PI). This proof-of-concept study demonstrates that IRTI provides an objective evaluation of response to PBMT treatment and correlates with observed and measured clinical response.
\end{abstract}

Keywords: infrared thermal imaging, infrared thermography, veterinary thermal imaging, pain assessment, osteoarthritis, canine back pain, canine brief pain inventory, photobiomodulation therapy, laser therapy

\section{Introduction}

Infrared thermal imaging (IRTI) provides a noncontact, noninvasive, accurate, and quantifiable graphic of the temperatures being radiated from the skin of the patient. The radiated energy correlates directly with blood flow beneath the skin [1-4]. Normal patients are thermally symmetrical when comparing contralateral anatomical areas $[5,6]$. Thermal asymmetry in contralateral anatomical areas results from an increase in underlying circulation caused by inflammation, infection, or neoplasia, or a decrease in circulation resulting from reduced sympathetic innervation of the area. [7-18].

IRTI has been used in clinical veterinary applications for nearly 60 years. The first research paper assessing clinical veterinary applications of IRIT was published in 1964 [19]. Multiple publications have validated clinical veterinary use of IRTI [10, 20-32]. Veterinary-specific guidelines were established in 2019 [33]. 
Photobiomodulation therapy (PBMT) delivers visible or near-infrared photons that produce a biochemical cascade of events in cells and tissues. Photobiomodulation induces autocrine signaling within cells, resulting in modulation of cell physiology and function and paracrine cell-to-cell signaling resulting in modulation of tissue physiology and function. Photoreceptor molecules within the cell absorb photonic energy resulting in increased release of adenosine triphosphate, reactive oxygen species, and nitric oxide. These bioactive substances incite a biochemical cascade of events that lead to increased circulation, reduced pain, modulation of inflammation, and acceleration of healing [3443].

The use of PBMT for treatment of pain and inflammation associated with neuromusculoskeletal disorders and osteoarthritis has been well documented in multiple species [44-49].

IRTI objectively measures radiant temperature data, displays the data visually, and allows physiological measurement of the patient's response to PBMT.

\section{Methods}

\subsection{Qualifying Participants}

Study participants were patients of a rehabilitation center that met the inclusion criteria of a history of back pain, short to medium haircoat, body weight of $15-55 \mathrm{~kg}$., and no current dermatological disorders.

To be included, the patient's initial physical exam had to reveal palpable back pain along the thoracic and/or lumbar spine in the epaxial and paravertebral muscles. Preference was given to patients with diagnostic evidence of age-related degenerative osteoarthritis of the thoracic and/or lumbar spine. The attending veterinarian for each participant prescribed PBMT of the musculature of the back. Any previously prescribed pharmaceutical, nutraceutical, or holistic treatment protocols were maintained during the study.

Participants could not receive PBMT, extracorporeal shock wave therapy (ESWT), or transcutaneous pulsed electromagnetic field therapy (tPEMF) for seven days prior to entering the study and could not receive ESWT or tPEMF at any time during the study. Participants had to discontinue any underwater treadmill sessions, therapeutic exercises, or massage techniques for four days prior to and during the study.

Owners were given a summary of the design and intent of the study and required to sign a participant release. The release included the provision that patients would be withdrawn from the study if examination revealed continuation might have any negative effects on the patient.

\subsubsection{Initial Patient Evaluation for Inclusion in the Study}

Initial clinical evaluation of each qualifying participant included a patient history, currently in-place therapeutic modalities, physical exam, body condition score (BCS), CSU-CCPS, and owner completion of a CBPI-PS and CBPI-PI worksheet. A dorsal IRTI of the back was taken framing from T6-7 to S1-3, including all paravertebral epaxial muscles, to provide baseline temperature data.

\subsection{Study Design}

\subsubsection{Day One}


The patient was given another physical examination followed by time to equilibrate to the ambient room temperature of temperature of $20^{\circ} \mathrm{C}$. BCS and CSU-CCPS were recorded, and the owner completed a CBPI.

A dorsal IRTI of the back was taken framing from T6-7 to S1-3, including all paravertebral epaxial muscles. The thermal imaging system used has a $640 \times 512$ high-resolution medical-grade detector with a $<.02^{\circ} \mathrm{C}$ sensitivity and accuracy of $+/-1^{\circ} \mathrm{C}$. Temperature data was collected from 327,680 points and compiled using calibrated veterinaryspecific software (Digatherm IR 640, Infrared Cameras Inc, Beaumont, TX, United States).

In each IRT image, an anatomical area from T6-7 to S1-3 including all paravertebral epaxial musculature, was defined as the region of interest (ROI). This ROI also defined the treatment area for PBMT in each patient.

PBMT was administered at a fluence of 20 joules $/ \mathrm{cm}^{2}$, using continuously delivered blended wavelengths of 650,810, and $980 \mathrm{~nm}, 10$ watts of power, spot size $4.91 \mathrm{~cm}^{2}$, and power density of $2.04 \mathrm{~W} / \mathrm{cm}^{2}$ (Companion Therapy Laser CTX-15, LiteCure, Wilmington, DE, United States). The delivery handpiece was kept in-contact with the hair and skin and moved uniformly over the ROI. All IRTI imaging and PBMT treatments were performed by the same person on each patient throughout the study.

The PBMT treatment parameters and application protocol were similar to those previously reported for treatment of osteoarthritis and neuromusculoskeletal disorders in dogs [48-50]. Information about device specifications, application method, and treatment parameters previously established as important is in Tables 1 [51].

Table 1: Photobiomodulation Treatment Parameters

\begin{tabular}{|c|c|c|c|}
\hline \multicolumn{4}{|c|}{ Photobiomodulation Therapy Device Information } \\
\hline \multicolumn{4}{|c|}{ Manufacturer: Companion Animal Health } \\
\hline \multicolumn{4}{|c|}{ Model: CTX-15 } \\
\hline \multicolumn{4}{|c|}{ Year Manufatured: 2018} \\
\hline \multicolumn{4}{|c|}{ Number of Emitters: One } \\
\hline \multicolumn{4}{|c|}{ Beam Delivery: Fiberoptic, On-contact, Rollerball Handpiece } \\
\hline \multicolumn{4}{|c|}{ Irradiation Parameters } \\
\hline \multicolumn{4}{|c|}{ Wavelength/Beam Percentage: 980 nm/57\%, 810 nm/38\%, 650 nm/5\% } \\
\hline \multicolumn{4}{|c|}{ Operation Mode: CW } \\
\hline \multicolumn{4}{|c|}{ Power Output: 10W (+/-5\%) } \\
\hline \multicolumn{4}{|c|}{ Spot Size: $4.91 \mathrm{~cm}^{2}$} \\
\hline \multicolumn{4}{|c|}{ Beam Shape: Circular } \\
\hline \multicolumn{4}{|c|}{ Fluence: $20 \mathrm{~J} / \mathrm{cm}^{2}$} \\
\hline \multicolumn{4}{|c|}{ Application technique: On-contact,light pressure, scanned uniformly over the ROI at 3-5cm/sec. } \\
\hline$\underline{\text { Patient }}$ & $\underline{\text { Total Area (sq. } \mathrm{cm})}$ & $\underline{\text { Total Time (minutes) }}$ & $\underline{\text { Total Joules Administered }}$ \\
\hline 1 & 672 & 22.4 & 13440 \\
\hline 2 & 260 & 8.7 & 5200 \\
\hline 3 & 360 & 12 & 7200 \\
\hline 4 & 384 & 12.8 & 7680 \\
\hline 5 & 480 & 16 & 9600 \\
\hline 6 & 456 & 15.2 & 9120 \\
\hline
\end{tabular}




\begin{tabular}{cccc}
\hline 7 & 264 & 8.8 & 5280 \\
\hline 8 & 588 & 19.6 & 11760 \\
\hline 9 & 560 & 18.7 & 11200 \\
\hline 10 & 270 & 9 & 5400 \\
\hline 11 & 532 & 17.7 & 10640 \\
\hline 12 & 456 & 15.2 & 9120 \\
\hline
\end{tabular}

\subsubsection{Days 2, 3, and 4}

On days 2, 3, and 4 PBMT was administered using the same protocol and delivery as the day 1 treatment.

\subsubsection{Day 7}

The patient was given a physical examination followed by time to equilibrate to the ambient room temperature. The owner was asked "Compared to yesterday, is your pet the same, better or worse?" A dorsal IRTI was taken of the patient's back, using the same framing as previous images.

\subsection{Data Collection}

On day 0 and 7 data was collected from each participant. This data included the thermographic measurement of the minimum, maximum, and average temperature in each ROI, a CSU-CCPS, an owner assessment of improvement or no improvement using a "better", "worse", or "same," an owner generated Canine Brief Pain Inventory (CBPI) worksheet and an owner evaluation of the quality of life (QOL) using a scale of "poor", "fair", "good", "very good", or "excellent". For each patient, the changes in temperatures in the region of interest (minimum, average and maximum) ( $\Delta$ Tmin, $\Delta$ Tave, $\Delta \operatorname{Tmax}$ ) for days 0 and 7 were calculated. These calculations were performed by the thermal imaging device software.

\subsection{Statistical Analysis}

Descriptive statistics were calculated. Normally distributed continuous variables were expressed as mean and standard deviation and non-normal distributed variables were expressed as median and range. To determine the correlation between two variables (ROI $\Delta \mathrm{T}$ and CBPI and ROI $\Delta \mathrm{T}$ and CSU-CCPS) for each subject who received photobiomodulation, the Mann-Whitney U-test was used to compare the change in CSUCCPS, CBPI-PS and CBPI-PI scores with the change in ROI thermal temperatures from day 0 to day 7. Two-tailed assessments were used and $P$ values $<.05$ were considered significant. All analyses were performed using a statistical program (IBM SPSS Statistics for Windows, Version 25.0, IBM Corp, Armonk, New York).

\section{Results}

\subsection{Patients}

Twelve dogs meeting the inclusion and exclusion criteria participated in the trial. All patients completed day 0 and day 7 pain assessment. All twelve dogs had a history of generalized lower back pain.

Four of the twelve had a diagnosis of chronic back pain combined with multi-joint osteoarthritis. One dog had a known adrenal tumor. Two dogs had a history of ataxia 
and weakness. Although the laser therapist was not blinded to which animals had multiple joint osteoarthritis, there was no attempt to provide PBMT to anywhere on the patient other than the specified ROI (in this case the thoracolumbar epaxial muscles.) All owners completed the CBPI on days 0 and 7. All dogs completed thermography studies specifically as outlined. Baseline characteristics of all 12 dogs are presented in Table 2.

Table 2: Baseline characteristics of 12 dogs with non-specific back pain enrolled in an open study evaluating the correlation between pain scores, thermal data, and response to PBMT.

\begin{tabular}{ll} 
Number of participants: & 12 \\
\hline Age in years median (range): & $12(7-15)$ \\
\hline Weight in kg median (range): & $24.1(7.7-33.5)$ \\
\hline Body Condition Score (BCS/10) median (range): & $5.5(4-7)$ \\
\hline Pain Severity Score Day 0 median (range): & $4.625(2.0-7.0)$ \\
\hline Pain Interference Score Day 0 median (range): & $5.835(2.67-7.33)$ \\
\hline CSU-CCPS Score Day 0 median (range): & $1.5(0.75-3.0)$ \\
\hline
\end{tabular}

\subsection{Qualitative analysis of IRTIs and correlation to Pain Scores}

For each dog, the IRTIs were paired - with days 0 and 7 presented on the same page. Three veterinarians, experienced with IRTI, and blinded to patient signalment, history, and pain scores, evaluated the twelve pair of IRT images qualitatively and recorded IRTI improvement or no improvement on a scale of "better", "worse", or "same". These results were compared to the owner assessment of QOL on days 0 and 7. Any improvement of QOL was tallied as "better", any worsening of QOL was reported as "worse" and no change in QOL was reported as "same". Figure 1 includes paired IRT images of three patients for qualitative interpretation. 


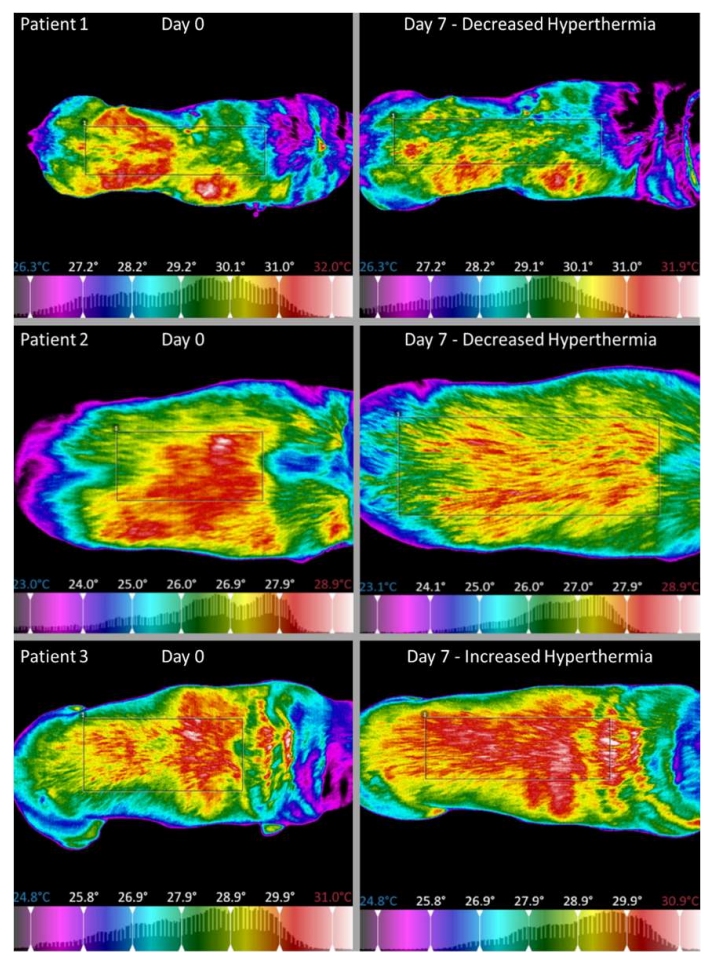

Figure 1: Paired IRT images (Day 0 vs Day 7) of three patients for qualitative interpretation. Patients 1 and 2 show qualitatively decreased hyperthermia in the region of interest. Patients 3 shows qualitatively increased hyperthermia in the region of interest.

Of the 12 patients, while the paired IRT images of three patients showed a qualitative visual increase in hyperthermia, no owner reported that QOL was worse. Four paired IRT images were visually the same, and five owners reported that QOL was the same. Five paired IRT images showed a visual decrease in hypothermia, and seven owners reported improved QOL. The results are represented in Figure 2.

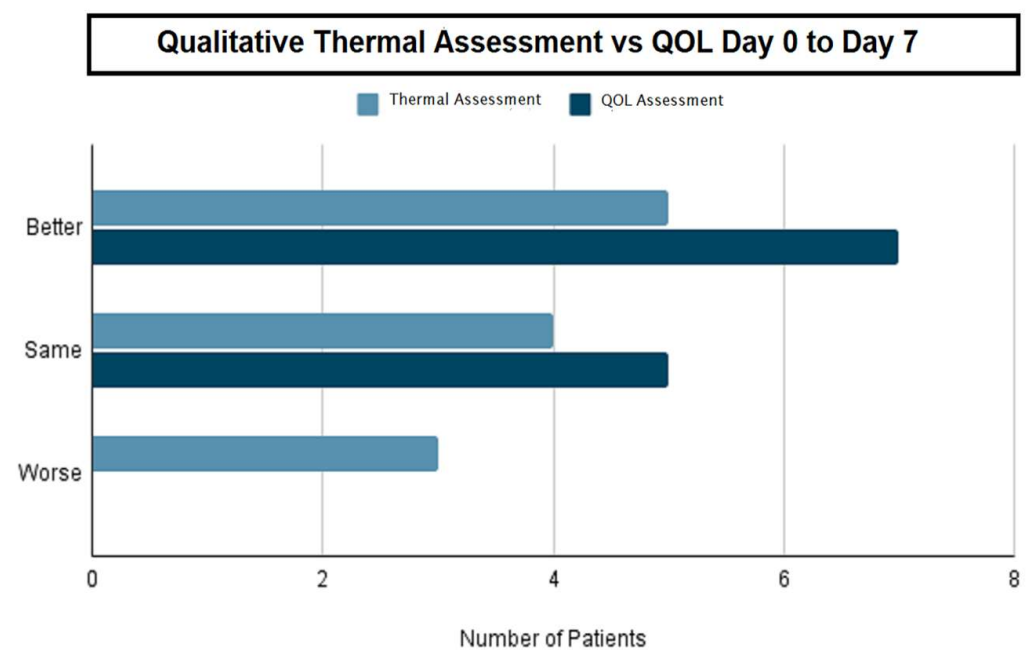

Figure 2: Qualitative Thermal Assessment versus QOL Day 0 to Day 7. 


\subsection{Ability of Pain Scores and IRTI ROI temperatures to detect response to Photobi- omodulation Therapy (PBMT)}

From day 0 to day 7, ten of the twelve dogs' clinician pain assessment scores improved as measured by the CSU-CCPS. The remaining two dogs CSU-CCPS scores stayed the same. The owner assessments performed on days 0 and 7 were more variable, with eight owners reporting improvement in CBPI-PS, and ten owners reporting improvement in CBPI-PI.

Each thermal image was evaluated to determine $\Delta \operatorname{Tmin}, \Delta$ Tave, and $\Delta \operatorname{Tmax}$ in the ROI between days 0 and 7 . Eight patients showed a decrease in $\Delta \operatorname{Tmin}, 6$ showed a decrease in $\Delta$ Tmax and 7 showed a decrease in $\Delta$ Tave. Median and mean results are shown in Table 3.

Table 3: Comparison of response to treatment outcomes using the owner-completed CBPI, the clinician-completed CSU-CCPS, and the change in ROI $\triangle \mathrm{T}$.

\begin{tabular}{cccccc}
\hline $\begin{array}{c}\text { Change in } \\
\text { CSU-CCPS }\end{array}$ & $\begin{array}{c}\text { Change in } \\
\text { CBPI Pain } \\
\text { Severity Score }\end{array}$ & $\begin{array}{c}\text { Change in } \\
\text { CBPI Pain } \\
\text { Interference } \\
\text { Score }\end{array}$ & $\begin{array}{c}\text { Change in } \\
\text { ROI } \Delta \text { Tmin }\end{array}$ & $\begin{array}{c}\text { Change in } \\
\text { ROI } \Delta \text { Tmax }\end{array}$ & $\begin{array}{c}\text { Change in } \\
\text { ROI } \Delta \text { Tave }\end{array}$ \\
\hline Median/Range & Median/Range & Median/Range & Mean+sd & Mean+sd & Mean+sd \\
\hline$-0.25(-1.25-0)$ & $-1.0(-3.5-0.75)$ & $-2.0(-4.67-0.67)$ & $-0.392 \pm 0.916$ & $0.142 \pm 0.797$ & $-0.225 \pm 0.926$ \\
\hline
\end{tabular}

The Mann-Whitney U-test showed a statistically significant correlation between all three $\Delta \mathrm{T}$ measurements and owner assessment of CBPI-PI $(\mathrm{P}=.01, .001, .008)$. Both the CBPI-PS and the CSU-CCPS showed statistically significant correlation only with $\triangle \mathrm{Tmax}$ $(\mathrm{P}=.03, .02)$. Table 4 summarizes the U-test results.

Table 4: Mann-Whitney U test Calculator to compare Pain Scores to ROI $\Delta \mathrm{T}$.

\begin{tabular}{|c|c|c|c|c|}
\hline & U value & Critical Value of $U$ at $p<.05$ & z score & p-value \\
\hline CBPI-PS vs $\Delta \mathrm{Tmin}$ & 53.5 & 42 & -1.03923 & .15 \\
\hline CBPI-PS vs $\Delta T \max$ & 37.5 & 42 & -1.96299 & .03 \\
\hline CBPI-PS vs $\triangle$ Tave & 47.5 & 42 & -1.38564 & .08 \\
\hline CBPI-PI vs $\Delta$ Tmin & 33 & 42 & -2.2228 & .01 \\
\hline CBPI-PI vs $\Delta \mathrm{Tmax}$ & 21 & 42 & -2.9156 & .001 \\
\hline CBPI-PI vs $\Delta$ Tave & 29.5 & 42 & -2.4249 & .008 \\
\hline CSU-CCPS vs $\triangle \mathrm{Tmin}$ & 70 & 42 & -0.0866 & .46 \\
\hline CSU-CCPS vs $\triangle T$ max & 36 & 42 & -2.0496 & .02 \\
\hline CSU-CCPS vs $\triangle$ Tave & 72 & 42 & 0.0289 & .49 \\
\hline
\end{tabular}


The relationship between improvement in CBPI-PS, CBPI-PI and improvement in ROI $\Delta$ Tmax is presented in Figure 3 . The lower left quadrant represents dogs with combined pain score improvements and corresponding $\Delta$ Tmax improvement. The upper right quadrant represents dogs with worsening pain scores and worsening ROI $\Delta$ Tmax.

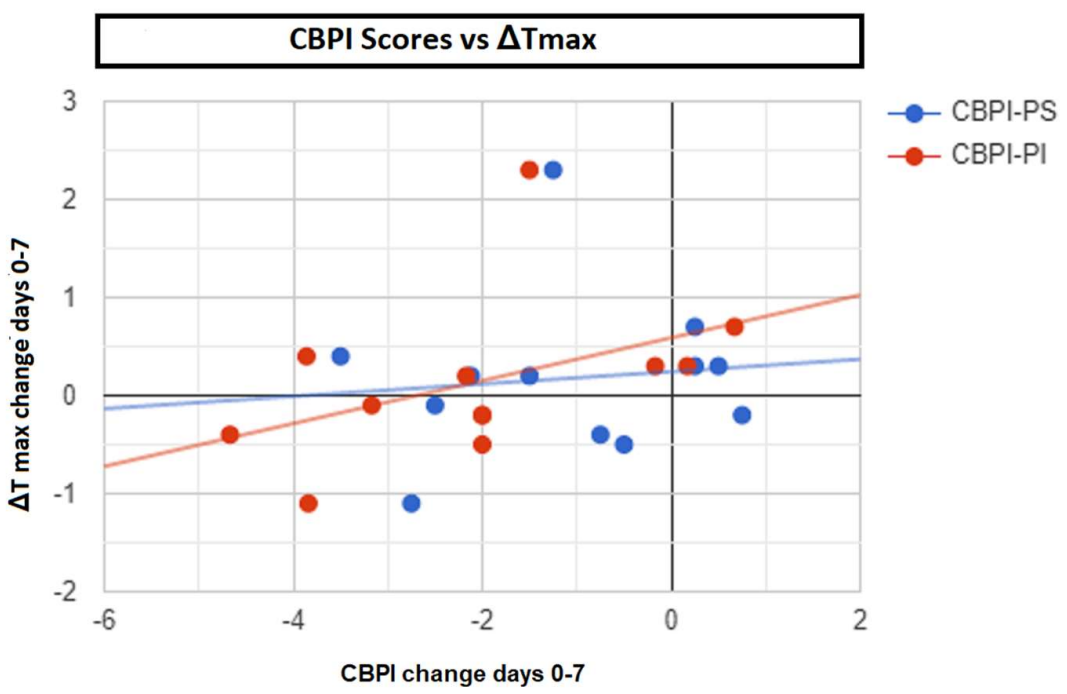

Figure 3: CBPI Scores versus $\triangle \mathrm{Tm}$ ax.

\section{Discussion}

Infrared thermography has long been used in various industries to measure minute changes in surface temperature otherwise invisible to the naked eye. Veterinary medicine offers a unique opportunity to explore thermography as a screening tool to aid the clinician in evaluating non-verbal patients. The use of IRTI in veterinary medicine has been reported for the evaluation of lameness in horses [52-54], companion animal clinical applications [10, 20-31], and most recently, structural screening in working dogs $[55,56]$. When combined with palpation, thermal imaging has been shown to be a useful tool in differentiating painful cats from non-painful cats [22]. However, to our knowledge, there has been no publication evaluating the correlation of thermography to clinician pain assessments or owner reported pain scoring after a particular treatment. Our objective in this proof-of-concept study was to determine if thermography is a more objective therapy outcome measurement than pain scoring alone.

Research studies that evaluate pharmacologic or non-pharmacologic pain treatment modalities often use pain scores along with an objective measurement to evaluate success or failure of treatment. For example, force plate gait analysis, combined with owner pain assessment, has been used to evaluate the efficacy of carprofen in dogs [57]. While pain scoring of non-verbal patients is difficult, its use is critical in providing adequate and successful pain management for the patient $[58,59]$. Subjective pain scores, such as numerical rating (NRS) or visual analogue scores (VAS) have been shown to have limited correlation with objective force plate data in dogs evaluated after knee surgery [60]. It is widely accepted that there is a significant caregiver placebo effect which can reach close to $40 \%$ in pet owners when compared to an objective outcome measurement and be even higher when veterinarians or veterinary staff perform pain assessment scoring 
[61]. Therefore, especially for chronic pain conditions, it is generally accepted practice to utilize a combination of pet owner scoring, objective measurement data, and clinician pain assessment to determine success or failure of a treatment protocol.

In this study, our objective measurement was temperature normalization over the assigned treatment area region of interest. Our results suggested that when we use an owner-reported, validated, canine chronic pain scoring tool, (CBPI), there was a statistically significant correlation between changes of temperature in the region of interest and the pain interference scores, while the pain severity scores were only significant when evaluating $\Delta \mathrm{Tmax}$.

We expected that $\Delta$ Tave measurements would be the most useful, however, in this sample population, $\Delta$ Tmax correlated best with CBPI scores. When collecting temperature data, all data points in the ROI are used to calculate the minimum, maximum, and average temperatures. The number of temperature data points at the highest temperatures, $\triangle T$ max, either increases or decreases as the circulation increases or decreases within that ROI. In this study, $\triangle \mathrm{Tmax}$ decreased in patients that responded to PBMT with a reduction in inflammation and a corresponding decrease in circulation.

Not surprisingly, the use of the most basic assessment tool, the owner QOL score, was the least accurate outcome measurement. Inherent bias in reporting is a continual problem in evaluation of treatment success [62] and our study reinforced the issue. It is interesting to consider whether sharing the Day 7 images with the pet owners would have changed their opinion of treatment outcome.

Pain is multifactorial, complex, and in all species of animals, can be influenced by biological, psychological, and social factors $[63,64]$. The successful use of any modality to treat pain can be difficult to assess as pain scoring and observational evaluation alone are often quite subjective. This study showed the addition of IRTI can remove some inherent bias and allow a more objective determination of outcome of treatment.

\section{Conclusions}

Infrared thermal imaging proved a useful tool for assessment of photobiomodulation treatment over time in canine patients with chronic generalized thoracolumbar back pain. Further investigation with a larger population of patients is necessary to assess the correlation between IRTI normalization of the treatment area, clinician pain scoring, and owner objective pain evaluation. This investigation proved the concept of IRTI as an objective means of monitoring response to therapy.

Data Availability Statement: The data presented in this study are available on request from the corresponding author. The data are not publicly available as they are part of the veterinary medical record

Author Contributions: Conceptualization and methodology, E.F., J.J., J.G., and R.R.; validation, J.J., J.G., and R.R.; formal analysis, J.J., J.G., and R.R.; investigation, E.F. and R.R; original draft preparation, review, and editing, J.J., J.G., and R.R. All authors have read and agreed to the published version of the manuscript.

Funding: This research received no external funding.

Informed Consent and Ethical Review: In the United States, there are no regulations or guidelines that specifically apply to private practices that wish to conduct research ${ }^{65}$. This study was performed in a private clinical practice, using client-owned animals, where a valid Veterinary-Client-Patient-Relationship (VCPR) existed. The therapy treatments and data collection described (PBMT, medical thermal imaging, and the use of pain score questionnaires) are treatments and 
interventions that are within the normal scope of routine veterinary practice and are considered standard of care.

Informed client consent was obtained from each owner, is certified in the manuscript, and a copy of the informed consent is attached for your review. The owners were fully informed that the research was to be published and there were no inherent risks to any data collection or treatment.

\section{References}

1. Sagaidachnyi, A.A.; Fomin, A.V.; Usanov, D.A.; Skripal, A.V. Thermography-based blood flow imaging in human skin of the hands and feet: a spectral filtering approach. Physiol Meas 2017 38(2), 272-288.

2. Tattersall, G.J. Infrared thermography: A non-invasive window into thermal physiology. Comp Biochem Physiol a Mol Integr Physiol 2016 202, 78-98.

3. Vainionpää, M.; Tienhaara, E.; Raekallio, M.; Junnila, J.; Snellman, M.; Vainio, O. Thermographic imaging of the superficial temperature in racing greyhounds before and after the race. Sci World Journ 2012, 1-6.

4. Casas-Alvarado, A.; Mota-Rojas, D.; Hernández-Ávalos, I.; Mora-Medina, P.; Olmos-Hernández, A.; Verduzco-Mendoza, A.; Reyes-Sotelo, B.; Martínez-Burnes, J. Advances in infrared thermography: Surgical aspects, vascular changes, and pain monitoring in veterinary medicine. J Therm Biol 2020 92, 102664.

5. Niu, H.; Lui, P.W.; Hu, J.S.; Ting, C.K.; Yin, Y.C.; Lo, Y.L.; Liu, L.; Lee, T.Y. Thermal symmetry of skin temperature: Normative data of normal subjects in Taiwan. Chin Med J 2001 64(8), 459-68.

6. Voloshyn, H.H. The thermal pattern of skin in healthy persons. Lik Sprava 2002 1, 20-24.

7. Purohit, R.C.; McCoy, M.D. Thermography in the diagnosis of inflammatory processes in the horse. Am J Vet Res 1980 41, 11671174.

8. Chudeca, M.; Lubkowska, A.; Leźnicka, K.; Krupecki, K. The use of thermal imaging in the evaluation of symmetry of muscle activity in various types of exercises (symmetrical and asymmetrical). J Hum Kinet 2015 30(49), 141-147.

9. Liu, X.L.; Hong, W.X.; Song, J.L.; Wu, Z.Y. Using infrared thermal asymmetry analysis for objective assessment of the lesion of facial nerve function. Guang Pu Xue Yu Guang Pu Fen Xi 2012 32(3), 647-650.

10. Grossbard, B.P.; Loughin, C.A.; Marino, D.J.; Marino, L.J.; Sackman, J.; Umbaugh, S.E.; Solt, P.S.; Afruz, J.; Leando, P.; Lesser, M.L.; Akerman, M. Medical Infrared Imaging (Thermography) of Type I Thoracolumbar Disk Disease in Chondrodystrophic Dogs. Vet Surg 2014 43(7), 869-876.

11. Ring,E.F.J.; Ammer, K. Infrared thermal imaging in medicine. Physiol. Meas 2012 17533(3), R33-46.1762.

12. Lahiri, B.B.; Bagavathiappan, S.; Jayakumar, T.; Philip. J. Medical applications of infrared thermography: A review. Infrared Phys Technol 2012 55(4), 221-235.

13. Campos-Nitrini, A.G.; Cogliati, B.; Matera, J.M. Thermographic assessment of skin and soft tissue tumors in cats. J Feline Med Surg 2021 23(6), 513-518.

14. Niu, H.H.; Lui, P.W.; Hu, J.S.; Ting, C.K.; Yin, Y.C.; Lo, Y.L.; Liu, L.; Lee, T.Y. Thermal symmetry of skin temperature: normative data of normal subjects in Taiwan. Chi Med. J 2001 64(8), 459-468.

15. Vardasca, R.; Plassmann, P.; Ring, F.; Jones, C.D. Thermal symmetry of the upper and lower extremities in healthy subjects. Thermol Int 2012 22(2), 53-60.

16. Alfieri, F.M.; Battistella, L.R. Body temperature of healthy men evaluated by thermography: A study of reproducibility. Technol Health Care 2018 26(3), 559-564.

17. Antonaci, F.; Rossi, E.; Voiticovschi-Iosob, C.; Volta, G.D.; Marceglia, S. Frontal infrared thermography in healthy individuals and chronic migraine patients: Reliability of the method. Cephalalgia 2019 39(4), 489-496.

18. Doesburg, F.; Smit, J.M.; Paans, W.; Onrust, M.; Nijsten, M.W.; Dieperink, W. Use of infrared thermography in the detection of superficial phlebitis in adult intensive care unit patients: A prospective single-center observational study. Plos One 2019 14(3), $\mathrm{e} 0213754$.

19. Smith, W.M. Applications of thermography in veterinary medicine. Ann NY Acad Sci 1964 121, 248-254.

20. Rekant, S.I.; Lyons, M.A.; Pacheco, J.M.; Arzt, J.; Rodriguez, L.L. Veterinary Applications of Infrared Thermography. Am J Vet Res 2016 77(1), 98-107.

21. Redaelli, V.; Tanzi, B.; Luzi, F.; Stefanello, D.; Proverbio, D.; Crosta, L.; Di Giancamillo, M. Use of Thermographic Imaging in Clinical Diagnosis of Small Animal: Preliminary Notes. Ann Ist Super Sanita 2014 50(2), 140-146.

22. Vainionpää, M.H.; Raekallio, M.R.; Junnila, J.J.T.; Hielm-Björkman, A.K.; Snellman, M.P.M.; Vainio, O.M. A Comparison of Thermographic Imaging, Physical Examination and Modified Questionnaire as an Instrument to Assess Painful Conditions in Cats. J Feline Med Surg 2013 15(2), 124-131.

23. Marino, D.J.; Loughin, C.A. Diagnostic Imaging of the Canine Stifle: A Review. Vet Surg 2010 39(3), $284-295$.

24. Infernuso, T.; Loughin, C.A.; Marino, D.J.; Umbaugh, S.E.; Solt, P.S. Thermal imaging of normal and cranial cruciate ligamentdeficient stifles in dogs. Vet Surg 2010 39(4), 410-417.

25. McGowan, L.; Loughin, C.A.; Marino, D.J.; Umbaugh, S.E.; Liu, P.; Amini, M.; Solt, P.; Lesser, M.L.; Akerman, M. Medical Infrared Imaging of Normal and Dysplastic Elbows in Dogs. Vet Surg 2015 44(7), 874-882 
26. Garcia, E.F.V.; Loughin, C.A.; Marino, D.J.; Sackman, J.; Umbaugh, S.E.; Fu, J.; Subedi, S.; Lesser, M.L.; Akerman, M.; Schossler, J.E.W. Medical Infrared Imaging and Orthostatic Analysis to Determine Lameness in the Pelvic Limbs of Dogs. Open Vet J 2017 7(4), 342-348.

27. Waddell, R.E.; Marino, D.J.; Loughin, C.A.; Tumulty, J.W.; Dewey, C.W.; Sackman, J. Medical infrared thermal imaging of cats with hyperthyroidism. Am J Vet Res 2015 76(1), 53-59.

28. Pouzot-Nevoret, C.; Barthélemy, A.; Goy-Thollot, I.; Boselli, E.; Cambournac, M.; Guillaumin, J.; Bonnet-Garin, J.M.; Allaouchiche, B. Infrared Thermography: A Rapid and Accurate Technique to Detect Feline Aortic Thromboembolism. J Feline Med Surg 2018 20(8), 780-785.

29. Pavelski, M.; Silva, DM.; Leite, N.C.; Junior, D.A.; de Sousa, R.S.; Guérios, S.D.; Dornbusch, P.T.; Infrared Thermography in Dogs with Mammary Tumors and Healthy Dogs. J Vet Intern Med 2015 29(6), 1578-1583.

30. Sung, J.; Loughin, C.A.; Marino, D.J.; Leyva, F.; Dewey, C.W.; Umbaugh, S.; Lesser, M. Medical infrared thermal imaging of canine appendicular bone neoplasia. BMC Vet Res 2019 15(1), 430.

31. Casas-Alvarado, A.; Mota-Rojas, D.; Hernández-Ávalos, I.; Mora-Medina, P.; Olmos-Hernández, A.; Verduzco-Mendoza, A.; Reyes-Sotelo, B.; Martínez-Burnes, J. Advances in infrared thermography: Surgical aspects, vascular changes, and pain monitoring in veterinary medicine. J Therm Biol 2020 92, 10664.

32. Nitrini, A.G.C.; Cogliati, B.; Matera, J.M. Thermographic assessment of skin and soft tissue tumors in cats. J Feline Med Surg 2021 23(6), 513-518

33. American Academy of Thermology. Veterinary Guidelines for Infrared Thermography. Available online: https://aathermology.org/organization-2/guidelines/veterinary-guidelines-for-infrared-thermography/ (accessed on 26 October 2021).

34. Anders, J.J.; Kobiela-Ketz, A.; Wu, X. Basic Principles of Photobiomodulation and Its Effects at the Cellular, Tissue, and System Levels. In Laser Therapy in Veterinary Medicine: Photobiomodulation; Riegel, R.J., Godbold, J.C., Eds.; Wiley, Ames, United States, 2017; 36-51.

35. Cotler, H.B.; Chow, R.T.; Hamblin, M.R. The Use of Low-Level Laser Therapy (LLLT) For Musculoskeletal Pain. MOJ Orthop Rheumatol 2015 2(5), 00068.

36. Hamblin, M.R. (2017) Mechanisms and applications of the anti-inflammatory effects of photobiomodulation. AIMS Biophys 2017 4(3), 337-361.

37. Anders, J.J.; Arany, P.; Baxter, G.D.; Lanzafame, R. Light-Emitting Diode Therapy and Low-Level Light Therapy Are Photobiomodulation Therapy. Photobiomodul. Photomed. Laser Surg 2019 37(6), 325-326.

38. Yamada, E.F.; Dos Santos Stein, C.; Moresco, R.N.; Bobinski, F.; Palandi, J.; Fernandes, P.F.; Folmer, V.; da Silva, M,D. Photobiomodulation and Sida tuberculata combination declines the inflammation's markers in knee-induced osteoarthritis. Lasers Med Sci 2021 Jan 8. doi: 10.1007/s10103-020-03207-8. Online ahead of print.

39. Tomazoni, S.S.; Costa, L.O.P.; Joensen, J.; Stausholm, M.B.; Naterstad, I.F.; Leal-Junior, E.C.P.; Bjordal, J.M. Effects of photobiomodulation therapy on inflammatory mediators in patients with chronic non-specific low back pain: Protocol for a randomized placebo-controlled trial. Medicine (Baltimore) 2019 98(15), e15177.

40. Ramezani, F.; Neshasteh-Riz, A.; Ghadaksaz, A.; Fazeli, S.M.; Janzadeh, A.; Hamblin, M.R.; Mechanistic aspects of photobiomodulation therapy in the nervous system. Lasers Med Sci 2021 Feb 24. doi: 10.1007/s10103-021-03277-2. Online ahead of print.

41. de Souza Costa, M.; de Brito, T.V.; de Oliveira, S.B.; Souza Brauna, I.; Neto, J.C.R.M.; Teles, R.H.G.; Dutra, Y.M.; de Aguiar Magalhães, D.; Sousa, S.G.; de Sousa, J.A.; Branco, C.E.R.C.; Hazime, F.A.; Dos Reis Barbosa, A.L.; Vasconcelos, D.F.P.; Medeiros, J.V.R.; de Carvalho Filgueiras, M. Photobiomodulation exerts anti-inflammatory effects on the vascular and cellular phases of experimental inflammatory models. Lasers Med Sci 2021 Mar 31. doi: 10.1007/s10103-021-03298-x. Online ahead of print.

42. Dompe, C.; Moncrieff, L.; Matys, J.; Grzech-Leśniak, K.; Kocherova, I.; Bryja, A.; Bruska, M.; Dominiak, M.; Mozdziak, P.; Skiba, THI.; Shibli, J.A.; Volponi, A.A.; Kempisty, B.; Dyszkiewicz-Konwińska, M. Photobiomodulation-Underlying Mechanism and Clinical Applications. J Clin Med 2020 9(6), 1724.

43. Cronshaw, M.; Parker, S.; Arany, P. Feeling the Heat: Evolutionary and Microbial Basis for the Analgesic Mechanisms of Photobiomodulation Therapy. Photobiomodul Photomed Laser Surg 2019 37(9), 517-526.

44. Vallone, F.; Benedicenti, S.; Sorrenti, E.; Schiavetti, I.; Angiero, F. Effect of diode laser in the treatment of patients with nonspecific chronic low back pain: a randomized controlled trial. Photomed Laser Surg 2014 32(9), 490-494.

45. Hockman, L. Photobiomodulation Therapy in Veterinary Medicine: A Review. Top Companion Anim. Med 2018 33(3), 83-88.

46. Barale, L.; Monticelli, P.; Raviola, M.; Adami, C. Preliminary clinical experience of low-level laser therapy for the treatment of canine osteoarthritis-associated pain: A retrospective investigation on 17 dogs. Open Vet J 2020 10(1), 116-119.

47. Gendron, D.J.; Hamblin, M.R. Applications of Photobiomodulation Therapy to Musculoskeletal Disorders and Osteoarthritis with Particular Relevance to Canada. Photobiomodul Photomed Laser Surg 2019 37(7), 408-420.

48. Looney, A.L.; Huntingford, J.L.; Blaeser, L.L.; Mann, S. A randomized blind placebo-controlled trial investigating the effects of photobiomodulation therapy (PBMT) on canine elbow osteoarthritis. Can Vet J 2018 59(9), 959-966.

49. Miller, L.A.; Torraca, D.; De Taboada, L. Retrospective Observational Study and Analysis of Two Different Photobiomodulation Therapy Protocols Combined with Rehabilitation Therapy as Therapeutic Interventions for Canine Degenerative Myelopathy. Photobiomodul Photomed Laser Surg 2020 38(4), 195-205.

50. Piao, D.; Sypniewski, L.A.; Dugat, D.; Bailey, C.; Burba, D.J.; DeTaboada, L. Transcutaneous transmission of photobiomodulation light to the spinal canal of dog as measured from cadaver dogs using a multi-channel intra-spinal probe. Lasers Med Sci $201934(8), 1645-1654$. 
51. Jenkins, P.; Carroll, J. How to Report Low-Level Laser Therapy (LLLT)/Photomedicine Dose and Beam Parameters in Clinical and Laboratory Studies. Photomedicine and Laser Surg 2011 29(12), 785-7

52. Turner, T.A. Thermography as an Aid to the Clinical Lameness Evaluation. Vet Clin North Am Equine Pract 1991 7(2), $311-338$.

53. Weil, M.; Litzke, L.F.; Fritsch, R. Diagnostic Validity of Thermography of Lameness in Horses. Tierarztl Prax Ausg G Grosstiere Nutztiere 1998 26(6), 346-354.

54. Eddy, A.L.; Van Hoogmoed, L.M.; Snyder, J.R. The Role of Thermography in the Management of Equine Lameness. Vet J 2001 162(3), 172-181.

55. Alves, J.C.; Santos, A.; Jorge, P.; Lavrador, C.; Miguel Carreira, L. Clinical and diagnostic imaging findings in police working dogs referred for hip osteoarthritis. BMC Vet Res 2020 16(1), 425.

56. Alves, J.C.; Santos, A.; Jorge, P.; Lavrador, C.; Miguel Carreira, L. Evaluation of digital thermography imaging to assess and monitor treatment of police working dogs with naturally occurring hip osteoarthritis. BMC Vet Res 2021 17, 180.

57. Brown, D.C.; Boston, R.C.; Farrar, J.T. Comparison of force plate gait analysis and owner assessment of pain using the Canine Brief Pain Inventory in dogs with osteoarthritis. J Vet Intern Med 2013 27(1), 22-30.

58. McGuire, D.B.; Kaiser, K.S.; Haisfield-Wolfe, M.E.; Iyamu, F. Pain Assessment in Noncommunicative Adult Palliative Care Patients. Nurs Clin North Am 2016 51(3), 397-431.

59. Mathews, K.; Kronen, P.W.; Lascelles, D.; Nolan, A.; Robertson, S.; Steagall, P.V.; Wright, B.; Yamashita, K. Guidelines for recognition, assessment and treatment of pain: WSAVA Global Pain Council members and co-authors of this document. J Small Anim Pract 2014 55(6), E10-68.

60. Quinn, M.M.; Keuler, N.S.; Lu, Y.; Faria, M.L.; Muir, P.; Markel, M.D. Evaluation of agreement between numerical rating scales, visual analogue scoring scales, and force plate gait analysis in dogs. Vet Surg 2007 36(4), 360-367.

61. Conzemius, M.G.; Evans, R.B. Caregiver placebo effect for dogs with lameness from osteoarthritis. J Am Vet Med Assoc 2012 241(10), 1314-1319.

62. Gruen, M.E.; Dorman, D.C.; Lascelles, B.D.X. Caregiver placebo effect in analgesic clinical trials for cats with naturally occurring degenerative joint disease-associated pain. Vet Rec 2017 180, 473-473.

63. Bomholt, S.F.; Ballerup, D.; Harbuz, M.S.; Blackburn-Monro, G.; Blackburn-Monro, R.E. Involvement and role of the hypothalamo-pituitary-0adrenal (HPA) stress axis in animal models of chronic pain and inflammation. Stress 2004 7(1), 1-14.

64. Gatchel, R.J.; Peng, Y.; Peters, M.L.; Fuchs, P.N.; Turk, D.C. The Biopsychosocial Approach to Chronic Pain: Scientific Advances and Future Directions. Psychol Bull 2007 133, 581-624.

65. Baneux PJ, Martin ME, Allen MJ, Hallman TM. Issues related to institutional animal care and use committees and clinical trials using privately owned animals. ILAR J. 2014 55(1), 200-9. 